\title{
Gelesen und dabei gedacht...
}

\section{Werner Bauer}

Dr. med., Präsident des Schweizerischen Instituts für ärztliche Weiter- und Fortbildung SIWF

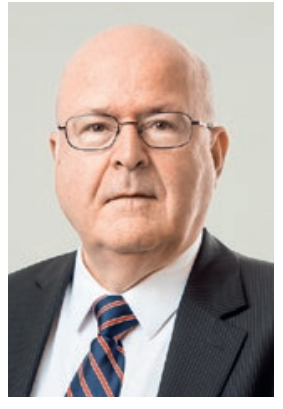

Vielleicht kann die eine Leserin oder der andere Leser meine immer wieder aufflammende Begeisterung für Sir William Osler nicht mehr ganz nachvollziehen oder sie sind gar daran, etwas Überdruss zu entwickeln, wenn ich der Versuchung einmal mehr nicht widerstehen kann, ihn zu zitieren [1]. Geboren wurde Osler 1849 in Kanada, lange Jahre war er als Internist in Philadelphia und als Gründungsprofessor an der Johns Hopkins Medical School in Baltimore tätig, 1919 starb er in Oxford. Ich kann schlicht nicht anders, als immer wieder einmal in der Literatur zu stöbern, die von ihm und über ihn geschrieben wurde, und da stosse ich regelmässig auf Perlen von Überlegungen, die damals treffend waren und bei denen sich auch zu heutigen Fragen bedenkenswerte Parallelen finden. Mit einigen Beispielen möchte ich die Überdrüssigen nicht strapazieren, aber allen anderen vor Augen führen, dass kluge Gedanken zeitlos und auch mit Blick auf die Covid-Pandemie noch erstaunlich treffend sein können.

Medicine is a science of uncertainty and an art of probability.

Wie kann das Wesen der Medizin noch prägnanter und zutreffender beschrieben werden? Die medizinische Wissenschaft muss mit Ungewissheit umgehen können - im neunzehnten Jahrhundert ebenso wie in Zeiten der Covid-Epidemie. Die Erwartungen, dass quälende offene Fragen kurzfristig mit unumstösslichen wissenschaftlichen Erkenntnissen beantwortet werden können, bleibt oft lange unerfüllt und ohne die Kunst des Abwägens von Wahrscheinlichkeiten geht es nicht.

Humanity has but three great enemies: Fever, famine and war; of these by far the greatest, by far the most terrible, is fever.

Je nach Weltregion trifft diese Aussage noch immer zu, ergänzt durch einen «vierten Feind», die Klimaveränderungen. Dass auch das Fieber in unseren Breitengraden wieder zur Bedrohung werden könnte, hat sich wohl bis zum Anfang dieses Jahres kaum jemand reell vorgestellt.

Soap and water and common sense are the best desinfectants.

Seife und Wasser haben ihre Bedeutung durchaus nicht verloren, obwohl wir im Moment allfällige Viren auf den Händen häufiger mit Alkohol und etwas Glyce- rin umzubringen versuchen. Über den Nutzen der Anwendung von "common sense» besteht wohl kein Zweifel, nur sind die Ansichten darüber, was er beinhaltet, kontrovers und Osler hat es leider verpasst, ihn genügend zu präzisieren.

Wherever plague exists an organized staff, an intelligent policy and a long purse are needed.

Die Pest kann man in diesem Satz mühelos durch die Covid-Epidemie ersetzen. Beim «organized staff» denkt man neben dem Spitalpersonal unter anderen heute an die Verantwortlichen für das «tracing». Die Kostenwelle rollt und sie wird lange nicht verebben. Auf die «intelligent policy» wollen wir hoffen; es wäre beruhigend, wenn es so etwas wie «evidence based politics» gäbe.

A sceptical attitude in these days of hasty observation and of still hastier conclusions is peculiarly appropriate. Diese Warnung scheint mir wieder ganz besonders berechtigt und aktuell, wenn man an die Flut von Prognosen, Hochrechnungen, Studienzwischenergebnissen, Impfstoffentwicklungsberichten und Hypothesen denkt, die alle mit dem Label "wissenschaftlich" auf Papier geflattert und über Bildschirme geflimmert kommen.

Es wäre klug und verantwortungsbewusst, wenn eloquente und mitteilungsfreudige Profi- und Hobby-Epidemiologen Oslers Empfehlung vor Interviews und «Primeurverlautbarungen» über Früh-, Erst- und Zwischenergebnisse von Studien beherzigen würden. Forschung muss sein, Hypothesen sind notwendig, Erstergebnisse müssen diskutiert und überprüft werden, aber den Entscheidungsträgern und der Bevölkerung ist mit einer «sceptical attitude» mehr gedient als mit dem Hinausposaunen von unbestätigten Teil- und Frühresultaten.

Damit sind wir bei der Presse und ihren Schwachpunkten angelangt. Sie setzen das Thema für Oslers Schlusswort zu diesem Artikel, das für sich selbst spricht: Believe nothing that you see in the newspapers - they have done more to create dissatisfaction than all other agencies. If you see anything in them that you know is true, begin to doubt it at once.

\footnotetext{
Literatur

1 M. Silverman, TJ Murray, CS Bryan (Herausgeber): The quotable Osler, ACP (2003).
} 